\title{
Code 7: An Automatic Pathway for Patients with Erectile Dysfunction After Prostate Surgery
}

Based on the results above it was felt that any pathway needed to be a standardized pathway of care for ED and also addressing side effects of therapy whilst managing patient expectations both pre and post operatively (Table 52.1).

- 'Proper aftercare needs to be in place.'-ID2

- 'If we are given a choice between cancer and erections, we choose clearance of cancer, but ED does need to be addressed.' - ID3.

This also extended to counselling. What is becoming quite apparent, is the requirement for psychosexual pathway as a recurrent theme. Whilst cancer control is acknowledged as initially important as part of survival, so too is ED.

Table 52.1 Code 7: Automatic pathway for ED

\begin{tabular}{l|l}
\hline & Patient quotes using Patient Identification Numbers \\
\hline $\begin{array}{l}\text { Requirement for } \\
\text { standardised pathway }\end{array}$ & 'The intervention needs to be automatic and standard \\
care.'-ID3
\end{tabular}

